Z. Klin. Chem. Klin. Biochem.

12. Jg. 1974 , S. 521-523

\title{
Eine einfache Methode zur Bestimmung von freiem und proteingebundenem Hämoglobin im Plasma
}

\author{
Von G. Weichert und $E$. Schulz \\ Aus der II. Medizinischen Klinik (Direktor: Prof. Dr. A. Kleinschmidt) der Medizinischen Hochschule Lübeck
}

(Eingegangen am 12. Februar/25. Juli 1974)

Im Rahmen experimenteller Untersuchungen zur glomerulokapillären Permeabilität der Hundeniere für Hämoglobin stellte sich das Problem eines einfachen Nachweises von freiem $\left(\mathrm{Hb}_{f}\right)$ und proteingebundenem $\left(\mathrm{Hb}_{b}\right)$ Hämoglobin im Plasma. Nachdem in Vorversuchen die Aussalzung des proteingebundenen Hämoglobins gesichert werden konnte, wurde folgendes Verfahren angewandt:

Hämoglobinhaltiges Plasma wurde mit Natriumsulfit $(280 \mathrm{~g} / 1)$ im Volumen-Verhältnis $1: 11$ für $3 \mathrm{~h}$ bei $37^{\circ} \mathrm{C}$ inkubiert. Die Proben wurden mit Diäthyläther überschichtet, kurz geschüttelt und für $10 \mathrm{~min}$ bei $2750 \mathrm{~g}$ zentrifugiert. Der Überstand wurde abgesaugt. Der Hämoglobingehalt der wäßrigen Phase $\left(\mathrm{Hb}_{\mathrm{f}}\right)$ sowie die Ausgangskonzentration des Plasmas an Hämoglobin $\left(\mathrm{Hb}_{\text {total }}\right)$ wurden mit der Hämiglobin-Cyanid-Methode gemessen. Die Konzentration des proteingebundenen Hämoglobins $\left(\mathrm{Hb}_{\mathrm{b}}\right)$ ergibt sich dann unter Berücksichtigung des Verdünnungsfaktors aus der Beziehung $\mathrm{Hb}_{\mathrm{b}}=\mathrm{Hb}_{\text {total }}-\mathrm{Hb}_{\mathrm{f}}$. Die methodischen Grenzen des Verfahrens werden diskutiert.

\section{A simple method for the determination of free and protein-bound haemoglobin in plasma}

In the course of experiments on the glomerulo-capillary permeability of dog kidney with respect to haemoglobin, a simple method was required for the measurement of free $\left(\mathrm{Hb}_{\mathrm{f}}\right)$ and protein-bound $\left(\mathrm{Hb}_{\mathrm{b}}\right)$ haemoglobin in the plasma. On the basis of the observation that the protein-bound haemoglobin can be separated by salting out, the following method was developed:

Plasma containing haemoglobin ( $\left.\mathrm{Hb}_{\text {total }}\right)$ was incubated for $3 \mathrm{hr}$ at $37^{\circ} \mathrm{C}$ with sodium sulphite solution $(280 \mathrm{~g} / \mathrm{l})$ in the volume ratio 1:11 respectively. After the addition of ether, samples were shaken briefly by hand, then centrifuged $(2750 \mathrm{~g}, 10 \mathrm{~min})$. The upper layer with the precipitated protein was removed by suction at the pump, and the concentrations of haemoglobin in the aqueous layer $\left(\mathrm{Hb}_{\mathrm{f}}\right)$ and in the starting plasma $\left(\mathrm{Hb}_{\text {total }}\right)$ were measured by the haemiglobin-cyanide method. Taking dilution factors in to account, the concentration of protein-bound haemoglobin is defined $\mathrm{as}_{\mathrm{Hb}}=\mathrm{Hb}_{\text {total }}-\mathrm{Hb}_{\mathrm{f}}$. Limitations of the method are discussed.

Intravasal freigesetżtes Hämoglobin $(\mathrm{Hb})$ wird vom Haptoglobin ( $\mathrm{Hp}$ ) des Plasmas im molaren Verhältnis 1:1 gebunden. Dabei ist freies, d. h. nicht proteingebundenes $\mathrm{Hb}$, im Plasma erst dann nachweisbar, wenn die Bindungskapazität des $\mathrm{H} p$ erschöpft ist (1). Die Glomeruluskapillaren der gesunden Niere sind für diesen Hämoglobin-Haptoglobbin-Komplex nicht durchlässig. Bei Bestimmung der renalen Hämoglobin-Clearance (2) ist es deshalb notwendig, den Anteil des proteingebundenen Hämoglobins $\left(\mathrm{Hb}_{b}\right)$ am gesamiten Hämoglobingehalt $\left(\mathrm{Hb}_{\text {total }}\right)$ des Plasmas zu ermitteln. Die bisherigen Veröffentlichungen zur Hämoglobinbestimmung basieren überwiegend auf der Anwendung der PeroxidaseReaktion. Zumeist wird der quantitative Nachweis mit dem Elektrophorese-Verfahren kombiniert, was jedoch eine geeignete apparative Eịnịchtung voraussetzt (3-9).

Im Rahmen eigener Untersuchungen zur glomerulokapillären Permeabilität der Hundeniere für Hämoglobin stellte sich das Problem eines einfachen und ohne großen apparativen Aufwand zu handhabenden, quantitativen Nachweises von proteingebundenem und freiem Plasmahämoglobin.

\section{Methodik}

Versuchstiere waren Bastardhunde von ; ,5 bis 39,0 kg Körpergewicht. Die Tiere erhielten mindestens eine Woc:!: lang natriumarme Reisdiät mit einer täglichen Eiweißzufuhr von 1,0 bis $1,5 \mathrm{~g} / \mathrm{kg}$ Körpergewicht. Flüssigkeitszufuhr nach Bedurf.

\section{Hämoglobinlösung}

Dem Versuchstier durch Venenpunktion entnommenes Blut wurde heparinisiert und $10 \mathrm{~min}$ bei $2750 \mathrm{~g}$ zentrifugiert. Das Zellsediment wurde dreimal mit physiologischer $\mathrm{NaCl}-\mathrm{Lösung}$ gewaschen und durch bidest. Wasser sowie Einfrieren bei $-20^{\circ} \mathrm{C}$ hämolysiert. Nach dem Auftauen wurde das Hämolysat 45 min bei $23000 \mathrm{~g}$ zentrifugiert. Es folgte die kryometrische Einstellung auf $300 \mathrm{mosmol} / 1$ durch Natriumchloridzugabe, sowie bei Verwendung zur Infusion - auf $50 \mathrm{~g} \mathrm{Hb} / 1$ (Kontrolle mit der Hämiglobin-Cyanid-Methode, (10-13)).

\section{Quantitative Aussalzung von $\mathrm{Hb}_{b}$ (Hb: Hp-Komplex)}

Hämolysat $(58 \mathrm{~g} \mathrm{Hb} / \mathrm{l})$ wurde mit autologem Plasma in einem Volumen-Verhältnis versetzt, daß sich $\mathrm{Hb}-K o n z e n t r a t i o n e n$ von 2,$35 ; 3,55 ; 6,35$ und $8,75 \mathrm{~g} / 1$ ergaben, und nachfolgend für $1 \mathrm{~h}$ bei $37^{\circ} \mathrm{C}$ inkubiert. Danach wurden die Proben $(0,5 \mathrm{ml})$ sämtlicher $\mathrm{Hb}$-Konzentrationen mit Natriumsulfitlösung (Merck Art. Nr. 6657) ansteigender Konzentration $(200 ; 250$ und $280 \mathrm{~g} / \mathrm{l})$ im Volumen-Verhältnis 1:11 versetzt und weitere $3 \mathrm{~h}$ inkubiert. Anschließend wurden die Inkubate $(5,5 \mathrm{ml}) \mathrm{mit} 1 \mathrm{ml}$ Diäthyläther überschichtet, kurz geschüttelt und für $10 \mathrm{~min}$ bei $2750 \mathrm{~g}$ zentrifugiert. Der Überstand wurde mit der Wasserstrahl- 
pumpe abgesaugt und der Hämoglobingehalt der wäßrigen Phase mit der Hämiglobin-Cyanid-Methode bestimmt. Zwischen Zugabe der Transformationslösung und Photometrieren (Zeiss Spektrophotometer) wurde ein zeitlicher Abstand von mindestens 30 min eingehalten. Der Eiweißgehalt der Proben vor und nach Natrium-Sulfit-Zugabe wurde mit Hilfe der Biuretreaktion gemessen (15).

\section{Infusionsexperimente}

Die Experimente wurden in Narkose mit Pentobarbital-Natrium ("Nembutal") vorgenommen, welche durch intravenöse Zufuhr von $10 \mathrm{mg} / \mathrm{kg}$ Körpergewicht eingeleitet wurde. Den Tieren wurden zunächst $4 \mathrm{~g}$ autologes Hämoglobin innerhalb $20 \mathrm{~min}$, anschließend $30 \mathrm{mg} \mathrm{Hb} / \mathrm{min}$ für die Dauer einer Stunde intravenös infundiert. Es folgte die Gabe von $2 \mathrm{~g} \mathrm{Hb} / 10 \mathrm{~min}$ und die Fortführung der Erhaltungsinfusion $(30 \mathrm{mg} \mathrm{Hb} / \mathrm{min}$ ) für mindestens eine weitere Stunde. Die Blutproben wurden durch intravenöse Punktion entnommen, sofort heparinisiert und bei $2750 \mathrm{~g}$ für $10 \mathrm{~min}$ zentrifugiert. $0,5 \mathrm{ml}$ Plasma wurden mit $5 \mathrm{ml}$ Natriumsulfit (Konz. $280 \mathrm{~g} / \mathrm{l}$ ) versetzt und die Proben im weiteren Arbeitsgang wie oben angegeben behandelt.

Die elektrophoretische Fraktionierung der Proben erfolgte mittels "Elphor"-Gerät, 200 V, 45 min, Acetatfolie, Phosphatpuffer: Ionenstärke $0,05, \mathrm{pH} 7,0$. Färbung mit $o$-Dianisidin und Wasserstoffperoxid (6).

\section{Ergebnisse}

Der Tabelle 1 ist zu entnehmen, daß die Aussalzquoten des Hämoglobins $(\Delta \mathrm{Hb})$ mit der Konzentration der verwandten Natriumsulfitlösungen ansteigen und schließlich bei einer Lösung von $280 \mathrm{~g} / \mathrm{l}$ Natriumsulfit für den Mittelwert $\bar{x}$ den Betrag 1,01 $\pm 0,05$ g/l erreichen. Die Aussalzverluste des Eiweißes (Globuline) zeigen die gleiche Tendenz und erreichen schließlich einen Mittelwert $\overline{\mathrm{x}}$ von $31 \pm 3 \mathrm{~g} / \mathrm{l}$.

In Abbildung 1 sind die Ergebnisse von 19 Infusionsexperimenten an 12 Hunden mit 126 Einzelmessungen wiedergegeben. Der Gesamthämoglobingehalt des Plasmas ( $\left.\mathrm{Hb}_{\text {total }}\right)$ wurde auf der Abszisse gegen die $\mathrm{zu}$ gehörigen Aussalzquoten an proteingebundenem Hämoglobin $\left(\mathrm{Hb}_{\mathbf{b}}\right)$ auf der Ordinate aufgetragen. Die Regressionsgerade $y=0,02 x+0,54$ läuft nahezu parallel zur Abszisse. Bei einem Korrelationskoeffizienten von

$$
r=0,084<r_{2 \alpha}=0,05, v=124=0,175
$$

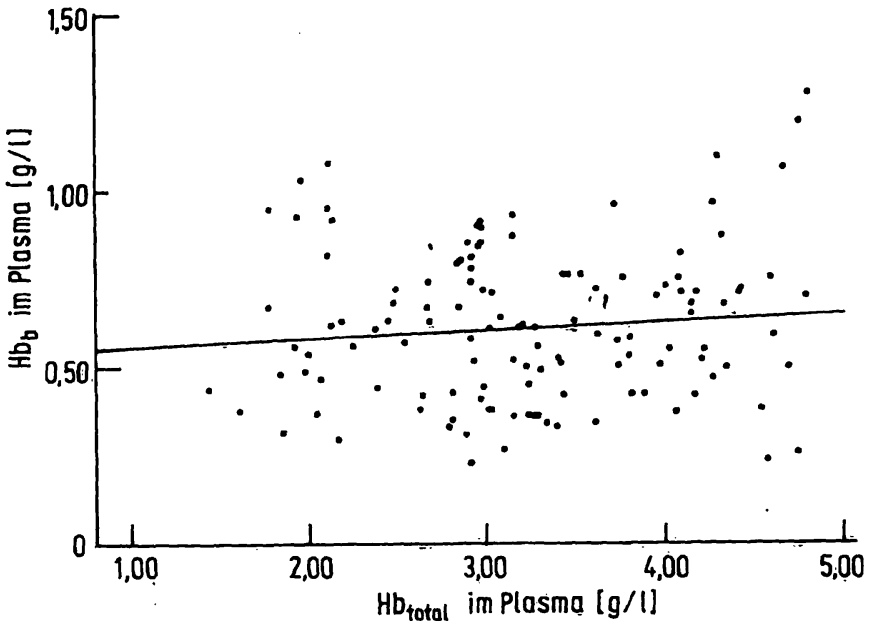

Abb. 1. Fehlende Korrelation żwischen der Konzentration des Gesamthämoglōbins $\left(\mathrm{Hb}_{\text {total }}\right)$ und des proteingebundenen Hämoglobins $\left(\mathrm{Hb}_{\mathrm{b}}\right)$. $\mathrm{n}=126$, Regression: $y=0,02 x+0,54 ; r=0,084$.

besteht keine signifikante Beziehung zwischen der Hämoglobinkonzentration der Plasmaproben $\left(\mathrm{Hb}_{\text {total }}\right)$ und den durch Aussalzung ermittelten Werten des proteingebundenen Hämoglobins $\left(\mathrm{Hb}_{\mathbf{b}}\right)$. Dabei ergeben sich keine neuen Gesichtspunkte, wenn man die Punktwolke aufschlüsselt und Regressionsgeraden für jedes Einzelexperiment berechnet.

Am Beispiel Abbildung 2 wurden im Rahmen eines Infusionsexperimentes zusammengehörige Proben von Plasma, Urin und sulfitfraktioniertem Plasma $(280 \mathrm{~g}$ Natriumsulfit/l) unter Anwendung der Methode vón Colfs und Verheyden (6) elektrophoretisch aufbereitet. Der Abbildung ist zu entnehmen, daß sich im Plasma sowohl freies als auch proteingebundenes Hämoglobin nachweisen lassen, während im Urin und sulfitfraktionierten Plasma nur freies Hämoglobin zur Darstellung kommt.

Tab. 1. Hämoglobinhaltiges Plasma: Aussalzung von proteingebundenem Hämoglobin und Globulinen durch Natriumsulfit.

$$
\begin{aligned}
& \mathrm{Hb}=\text { Hämoglobinkonzentration im Plasma (g/l), Nachweis: Hämiglobin-Cyanid-Methode } \\
& \Delta \mathrm{Hb}=\text { Aussalzquote des Hämoglobins (g/l) } \\
& \mathrm{E} \quad=\text { Eiweißkonzentration im Plasma (g/l), Nachweis: Biuretmethode } \\
& \Delta \mathrm{E}=\text { Aussalzquote des Eiweißes (Globuline) in } \mathrm{g} / \mathrm{l} \\
& \overline{\mathrm{x}} \quad=\text { Mittelwert der Aussalzquoten }
\end{aligned}
$$

\begin{tabular}{|c|c|c|c|c|c|c|c|c|c|c|c|c|c|}
\hline \multirow{2}{*}{\multicolumn{2}{|c|}{$\begin{array}{l}\text { Ausgangskonzen- } \\
\text { trationen }\end{array}$}} & \multicolumn{12}{|c|}{ Inkubation mit Natriumsulfit im Volumenverhältnis 1:11 } \\
\hline & & \multicolumn{4}{|c|}{$200 \mathrm{~g} / 1 \mathrm{Na}_{2} \mathrm{SO}_{3}$} & \multicolumn{4}{|c|}{$250 \mathrm{~g} / 1 \mathrm{Na}_{2} \mathrm{SO}_{3}$} & \multicolumn{4}{|c|}{$280 \mathrm{~g} / 1 \mathrm{Na}_{2} \mathrm{SO}_{3}$} \\
\hline $\begin{array}{l}\mathrm{Hb} \\
\mathrm{g} / \mathrm{l}\end{array}$ & $\begin{array}{l}\Delta \mathrm{E} \\
\mathrm{g} / \mathrm{l}\end{array}$ & $\begin{array}{l}\mathrm{Hb} \\
\mathrm{g} / \mathrm{l}\end{array}$ & $\underset{\mathrm{g} / \mathrm{l}}{\Delta \mathrm{Hb}}$ & $\begin{array}{l}E \\
g / 1\end{array}$ & $\begin{array}{l}\Delta \mathrm{E} \\
\mathrm{g} / 1\end{array}$ & $\begin{array}{l}\mathrm{Hb} \\
\mathrm{g} / \mathrm{l}\end{array}$ & $\begin{array}{l}\Delta \mathrm{Hb} \\
\mathrm{g} / 1\end{array}$ & $\begin{array}{l}E \\
g / 1\end{array}$ & $\begin{array}{l}\Delta \mathrm{E} \\
\mathrm{g} / \mathrm{l}\end{array}$ & $\begin{array}{l}\mathrm{Hb} \\
\mathrm{g} / 1\end{array}$ & $\begin{array}{l}\Delta \mathbf{H b} \\
\mathrm{g} / 1\end{array}$ & $\begin{array}{l}E \\
g / 1\end{array}$ & $\begin{array}{l}\Delta \mathrm{E} \\
\mathrm{g} / 1\end{array}$ \\
\hline $\begin{array}{l}8,75 \\
6,35 \\
3,55 \\
2,35\end{array}$ & $\begin{array}{l}85 \\
81 \\
77 \\
76\end{array}$ & $\begin{array}{l}8,52 \\
6,42 \\
3,30 \\
1,95\end{array}$ & $\begin{array}{r}0,23 \\
-0,07 \\
0,25 \\
0,40\end{array}$ & $\begin{array}{l}69 \\
61 \\
56 \\
53\end{array}$ & $\begin{array}{l}16 \\
20 \\
21 \\
23\end{array}$ & $\begin{array}{r}8,13 \\
5,72 \\
2,92 \\
1,60\end{array}$ & $\begin{array}{l}0,62 \\
0,63 \\
0,63 \\
0,75\end{array}$ & $\begin{array}{l}57 \\
50 \\
53 \\
43\end{array}$ & $\begin{array}{l}28 \\
31 \\
24 \\
33\end{array}$ & $\begin{array}{l}7,77 \\
5,37 \\
2,52 \\
1,32\end{array}$ & $\begin{array}{l}0,98 \\
0,98 \\
1,03 \\
1,03\end{array}$ & $\begin{array}{l}54 \\
52 \\
47 \\
42\end{array}$ & $\begin{array}{l}31 \\
29 \\
30 \\
34\end{array}$ \\
\hline$\overline{\mathbf{x}}$ & $(2 P=0,05)$ & & $\pm 0,31$ & & $20 \pm 5$ & & $66 \pm 0$ & & $29 \pm 6$ & & $1 \pm 0$ & & $31 \pm 3$ \\
\hline
\end{tabular}




Plasma Urin $\quad \begin{aligned} & \text { Plasmainkubat } \\ & \left(280 \mathrm{~g} /\left(\mathrm{Na}_{2} \mathrm{SO}_{3}\right)\right.\end{aligned}$

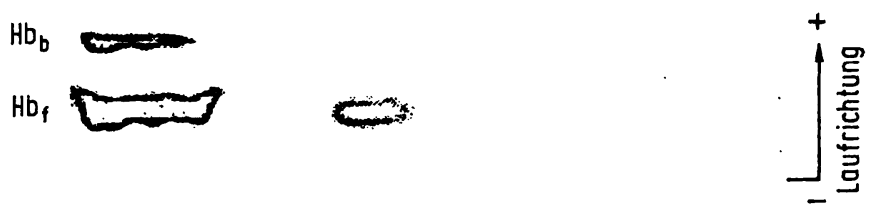

Abb. 2. Nachweis des proteingebundenen Hämoglobins im Plasma, jedoch nicht nach Sulfitausfällung und im Urin. Acetatfolien-Elektrophorese, Laufzeit $45 \mathrm{~min}$, Arbeitsspannung $200 \mathrm{~V}$, Peroxidase-Färbung mit $\sigma$-Dianisidin und Wasserstoffperoxid (6).

\section{Diskussion}

In Vorversuchen konnte unter Anwendung der Peroxidase-Reaktion (9) sichergestellt werden, daß proteingebundenes Hämoglobin mittels Natriumsulfit im Gegensatz zu wäßrig gelöstem Hämoglobin ausgesalzt werden kann.

Wenn man nun hämoglobinhaltiges Plasma mit Natriumsulfitlösungen ansteigender Konzentration inkubiert und die Hämoglobinkonzentration der zentrifugierten Inkubate mit Hilfe der Hämiglobin-Cyanid-Methode mißt, so ergibt sich - unabhängig von der vorgegebenen Hämoglobinkonzentration - eine zunehmende Aussalzquote proteingebundenen Hämoglobins. Dabei ist ein gleichgerichtetes Verhalten der übrigen Plasmaproteine bei Kontrolle durch die Biuretmethode zu beobachten. Bei Inkubation des hämoglobinhaltigen Plasmas mit einer auf $280 \mathrm{~g} / \mathrm{l}$ konzentrierten Natriumsulfitlösung erreicht man eine mittlere Eiweißaussalzquote von $31 \mathrm{~g} / 1$, ein Wert, welcher nach Richterich ((13), Mittelwert für Globulinaussalzung: $29 \mathrm{~g} / \mathrm{l})$ zu einer vollständigen Ausfällung der Globuline führt. Entsprechend ist

\section{Literaturverzeichnis}

1. Laurell, C.-B. \& Nyman, M. (1957), Blood 12, 493-506.

2. Weichert, G. \& Schulz, E. (1974), Nieren- und Hochdruckkrankheiten, $3,175-181$.

3. Lathem, W. (1959), J. Clin. Invest. 38, 652-658.

4. Faulstick, D. A., Lowenstein, J. \& Yiengst, M. J. (1962), Blood 20, 65-71.

5. Berggard, J. \& Bearn, A. G. (1962), Nature (London) 195, $1311-1312$.

6. Colfs, B. \& Verheyden, J. (1965), Clin. Chim. Acta 12, 470474.

7. Andersen, M. N., Mouritzen, C. V. \& Gabrieli, E. (1966), Ann. Surg. 163, 529-536.

8. Whittaker, M. (1968), Amer. J. Clin. Pathol. 5.0, 454-458. jetzt auch durch Elektrophorese im zentrifugierten Inkubat - selbst nach Anreicherung durch Ultrafiltration im Volumen-Verhältnis 1:10 - die Bande des HämoglobinHaptoglobinkomplexes nicht mehr nachweisbar.

Bezüglich der zur Hämoglobinbestimmung benutzten Hämiglobin-Cyanid-Methode ist zu bemerken, daß der Vorteil dieses Verfahrens gerade darin besteht, daß auch das nierengängige Hämi-Albumin erfaßt wird (13). Unter der Voraussetzung, daß zwischen Probezugabe und Messung ein Zeitraum von 30 min eingehalten wurde, ergab sich auch keinerlei Anhalt für eine Störung des Transformationsprozesses der Hämoglobin-Derivate in Hämiglobin-Cyanid. Schließlich besteht bezüglich der in-vivo-Versuche keine signifikante Korrelation zwischen der vorgegebenen Plasmakonzentration an Gesamthämoglobin und der Konzentration an proteingebundenem Hämoglobin, was eine Verfälschung der Aussalzquoten des Hämoglobin-Haptoglobin-Komplexes durch freie $\mathrm{Hb}$-Derivate und durch das Hämi-Albumin ausschließt.

Zusammenfassend erscheint uns das angewandte Verfahren zur differenzierten Bestimmung von freiem und proteingebundenem Hämoglobin durch die Ergebnisse der Aussalzversuche hinreichend begründet. Da die Elektrophoresestreifen aus apparativen Gründen nur qualitativ ausgewertet wurden, muß offen bleiben, ob noch geringe, unter praktischen Gesichtspunkten zu vernachlässigende Mengen des $\mathrm{Hb}: \mathrm{Hp}$-Komplexes in Lösung bleiben. Schließlich dürfte der bei Enteiweißung auftretende Volumenverdrängungseffekt (14) zu einer mindestens 5prozentigen Überhöhung der Werte des freien Hämoglobins fuihren. Die durch das Aussalzverfahren ermittelten Werte sind daher mit elektrophoretischen Befunden nur bedingt gleichzusetzen. Die Aussalzmethode gestattet jedoch den Vergleich von Werten, welche im Rahmen einer experimentellen Serie ermittelt wurden.
9. Mattenheimer, H. \& Adams, E. C. (1968), dies. Z. 6, 69-78.

10. Stadie, W. C. (1920), J. Biol. Chem. 41, 237-241.

11. Kampen, E. J. van, \& Zijlstra, W. G. (1961), Clin. Chim. Acta 6, 538-544.

12. Boroviczeny, G. G. (1964), Ery throcy tometric methods and their standardization, Karger, Basel/New York, N.Y., 1. Auflage, S. 1-124.

13. Richterich, R. (1968), Klinische Chemie, Akad. Verlagsges., Frankfurt (M), S. 341-343, 2. erweiterte Auflage.

14. Bürgi, W., Richterich, R. \& Mittelholzer, M. L. (1967), Klin. Wochenschr. 45, 83-86.

15. Weichselbaum, T. E. (1946), Amer. J. Clin. Pathol. 10, 40-49.
Dr. Günter Weichert II. Medizinische Klinik der Medizinischen Hochschule Lübeck 24 Lübeck

Ratzeburger Allee 160 